\title{
PAIRS OF ASTEROIDS PROBABLY OF A COMMON ORIGIN*
}

\author{
DAVID VOKROUHLICKÝ ${ }^{1}$ AND DAVID NESVORNÝ ${ }^{2}$ \\ ${ }^{1}$ Institute of Astronomy, Charles University, V Holešovičkách 2, CZ-18000 Prague 8, Czech Republic; vokrouhl@ cesnet.cz \\ ${ }^{2}$ Southwest Research Institute, 1050 Walnut St, Suite 300, Boulder, CO 80302, USA; davidn@ boulder.swri.edu \\ Received 2008 February 21; accepted 2008 April 30; published 2008 June 10
}

\begin{abstract}
We report the first observational evidence for pairs of main-belt asteroids with bodies in each pair having nearly identical orbits. The existence of $\sim 60$ pairs identified here cannot be reconciled with random fluctuations of the asteroid orbit density and rather suggests a common origin of the paired objects. We propose that the identified pairs formed by (i) collisional disruptions of km-sized and larger parent asteroids, (ii) Yarkovsky-O'Keefe-RadzievskiPaddack (YORP)-induced spin-up and rotational fission of fast-rotating objects, and/or (iii) splitting of unstable asteroid binaries. In case (i), the pairs would be parts of compact collisional families with many $\mathrm{km}$ - and sub-km-size members that should be found by future asteroid surveys. Our dynamical analysis suggests that most identified pairs formed within the past $\lesssim 1 \mathrm{Myr}$, in several cases even much more recently. For example, paired asteroids (6070) Rheinland and (54827) 2001 NQ8 probably separated from their common ancestor only 16.5-19 kyr ago. Given their putatively very recent formation, the identified objects are prime candidates for astronomical observations.
\end{abstract}

Key words: minor planets, asteroids

\section{INTRODUCTION}

The distribution of asteroid orbits across the main belt is uneven, reflecting the effects of various processes that shaped it over time. For example, dynamical resonances with planets depleted particular locations while, on the other hand, collisional breakups of large asteroids have created groups of asteroid fragments with similar orbits known as the asteroid families (Hirayama 1918). The standard method to identify an asteroid family is to search for concentrations of orbits in 3D space of proper elements: proper semimajor axis $a_{\mathrm{P}}$, proper eccentricity $e_{\mathrm{P}}$, and proper inclination $i_{\mathrm{P}}$ (Knežević et al. 2002). These elements, being more constant over time than the osculating orbital elements, provide a dynamical criterion that a group of asteroids has a common origin (see Bendjoya \& Zappalà 2002 and the references therein).

A different method can be used to identify asteroid families that formed recently (Nesvorný \& Vokrouhlický 2006; Nesvorný et al. 2006a). Instead of using the proper orbital elements, this new method relies directly on five osculating orbital elements: semimajor axis $a$, eccentricity $e$, inclination $i$, perihelion longitude $\varpi$, and nodal longitude $\Omega$. The very young families that formed in the last $\sim 1 \mathrm{Myr}$ show up as clusters in $5 \mathrm{D}$ space $(a, e, i, \varpi, \Omega)$, because fragments produced by a breakup have similar starting orbits and because they typically take $>1$ Myr before they can become dispersed by planetary perturbations and radiation forces. The clustering of fragments in mean anomaly $M$ is not expected due to the effects of Keplerian shear.

Here we report a new analysis of the distribution of asteroid osculating orbital elements that indicates that a large number of asteroid pairs exist in the main belt. The two asteroids in each identified pair have nearly identical osculating orbits. They may represent remnants of yet-to-be-characterized asteroid collisions, be parts of asteroids that underwent rotational fission and/or components of dissolved binaries. We explain the iden-

\footnotetext{
* The title paraphrases that of Hirayama's 1918 paper "Groups of asteroids probably of a common origin," where the first evidence was given for groups of asteroid fragments produced by disruptive collisions.
}

tification method of pairs in Section 2, discuss their statistical significance in Section 3, and estimate their formation times in Section 4. Selected pairs are discussed in Section 5. Different formation models are examined in Section 6.

\section{ASTEROID PAIRS}

We selected 369,516 asteroids from the AstOrb catalog (January 2008 release; Bowell et al. 1994) that have an observational arc longer than 10 days and $1.7<a<3.6 \mathrm{AU}$. This list was searched for asteroid pairs with unusually similar orbits. We defined the distance, $d$, in 5D space $(a, e, i, \varpi, \Omega)$ as

$$
\begin{aligned}
\left(\frac{d}{n a}\right)^{2}= & k_{a}\left(\frac{\delta a}{a}\right)^{2}+k_{e}(\delta e)^{2}+k_{i}(\delta \sin i)^{2}+k_{\Omega}(\delta \Omega)^{2} \\
& +k_{\varpi}(\delta \varpi)^{2},
\end{aligned}
$$

where $n$ is the mean motion, $(\delta a, \delta e, \delta \sin i, \delta \varpi, \delta \Omega)$ is the separation vector of neighboring bodies, and $\varpi$ and $\Omega$ are given in radians.

Following Zappalà et al. (1990), we used $k_{a}=5 / 4$ and $k_{e}=k_{i}=2$. We note that our results described below are insensitive to the exact values of coefficients $k_{a}, k_{e}$, and $k_{i}$ given that $k_{a} \sim k_{e} \sim k_{i} \sim 1$. The $k_{\Omega}$ and $k_{\varpi}$ values were chosen empirically. The results reported below were obtained with $k_{\Omega}=k_{\varpi}=10^{-5}$. We adopted these values rather than $k_{\Omega}=k_{\varpi}=10^{-6}$ of Nesvorný \& Vokrouhlický (2006) to impose smaller differences in angles for a given value of $d$.

To start with, we calculated distance $d$ from each of the 369,516 orbits to its nearest neighbor orbit. Figure 1 (black symbols labeled 1) shows the cumulative number of these orbit pairs, $N(<d)$, as a function of $d$. Figure 2 shows $N(<d)$ for the Hungaria region $(1.85<a<2.0 \mathrm{AU}, e<0.15$, and $15^{\circ}<i<25^{\circ}$ ). For $d \gtrsim 30 \mathrm{~m} \mathrm{~s}^{-1}$, the distributions follow a power law, $N(<d) \propto d^{\alpha}$, with an exponent $\alpha \approx 4$.7. Such a functional dependence is expected for a random distribution of points in 5D space where $N(<d) \propto d^{5}$. The small difference between the determined values of $\alpha$ and 5 partially stems from the slightly unequal weighting of different dimensions in Equation (1) (see Figure 1). 


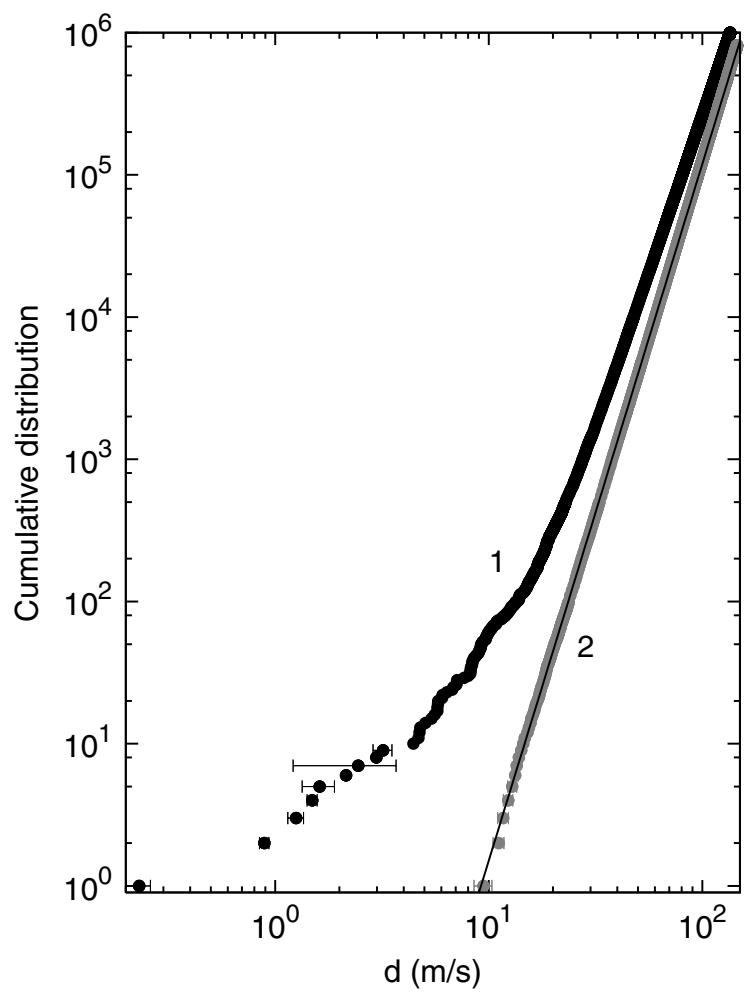

Figure 1. The cumulative number of pairs as a function of $d ; N(<d)$. The black symbols labeled 1 denote the distribution of real asteroids. The error bars associated with $d$ values of the ten tightest pairs were estimated by their orbit uncertainties. The gray symbols labeled 2 show the distribution obtained by selecting 370,000 test orbits with $1.7<a<3.6 \mathrm{AU}$. The associated error bars denote uncertainties determined from several such test distributions (see Section 4). The best-fit power law to blue symbols is $N(<d) \propto d^{4.91}$ (straight line).

The distribution in Figure 1 for $d \lesssim 20 \mathrm{~m} \mathrm{~s}^{-1}$ deviates from the expected dependence. It shows an excess of tight asteroid pairs with as many as 60 pairs with $d<10 \mathrm{~m} \mathrm{~s}^{-1}$. An excess of tight pairs is also apparent for $d<30 \mathrm{~m} \mathrm{~s}^{-1}$ in the Hungaria region (Figure 2). These results are puzzling and need closer inspection (see below). In Table 1, we list the osculating orbital elements of the eleven tightest pairs with $d<4.7 \mathrm{~m} \mathrm{~s}^{-1}$ and pair (6070) Rheinland-(54827) 2001 NQ8 with $d=$ $5.8 \mathrm{~m} \mathrm{~s}^{-1}$. The tightest pair with $d=0.23 \mathrm{~m} \mathrm{~s}^{-1}$ consists of two small Hungaria asteroids (63440) 2001 MD30 and 2004 TV14. The designations of asteroids in all identified pairs with $d<10 \mathrm{~m} \mathrm{~s}^{-1}$ are given in Table 2 . $^{3}$

We found that 15 pairs with $d<10 \mathrm{~m} \mathrm{~s}^{-1}$ are asteroids in known very-young asteroid families: five pairs in the Datura family (e.g., pair (1270) Datura and 2003 SQ168 with $d=$ $0.89 \mathrm{~m} \mathrm{~s}^{-1}$ ), five in the Karin cluster, two in Iannini, one in Veritas, one in Lucascavin, and one in Aeolia (Nesvorný et al. 2002, 2003, 2006a; Nesvorný \& Vokrouhlický 2006). These orbital regions are places with an extremely high number density of asteroid orbits where one is more likely to find tight pairs (see Section 3). However, these 15 pairs we found in the young families represent only $25 \%$ of the total. Therefore, the recent

\footnotetext{
3 In addition, we found one pair with $d<10 \mathrm{~m} \mathrm{~s}^{-1}$ in the Hilda population of resonant asteroids at $a \sim 3.9$ AU, namely (21930) 1999 VP61 and (22647) 1998 OR8 with $d=7.4 \mathrm{~m} \mathrm{~s}^{-1}$. This pair was excluded from the present analysis because its semimajor axis is outside the range considered in this work. We also found paired objects in the Kuiper belt. For example, Kuiper belt objects 2003 YN179 and 2004 OL12 have orbits with $d=13.4 \mathrm{~m} \mathrm{~s}^{-1}$. In these cases, however, the orbital uncertainty is generally large and makes a more thorough analysis difficult.
}

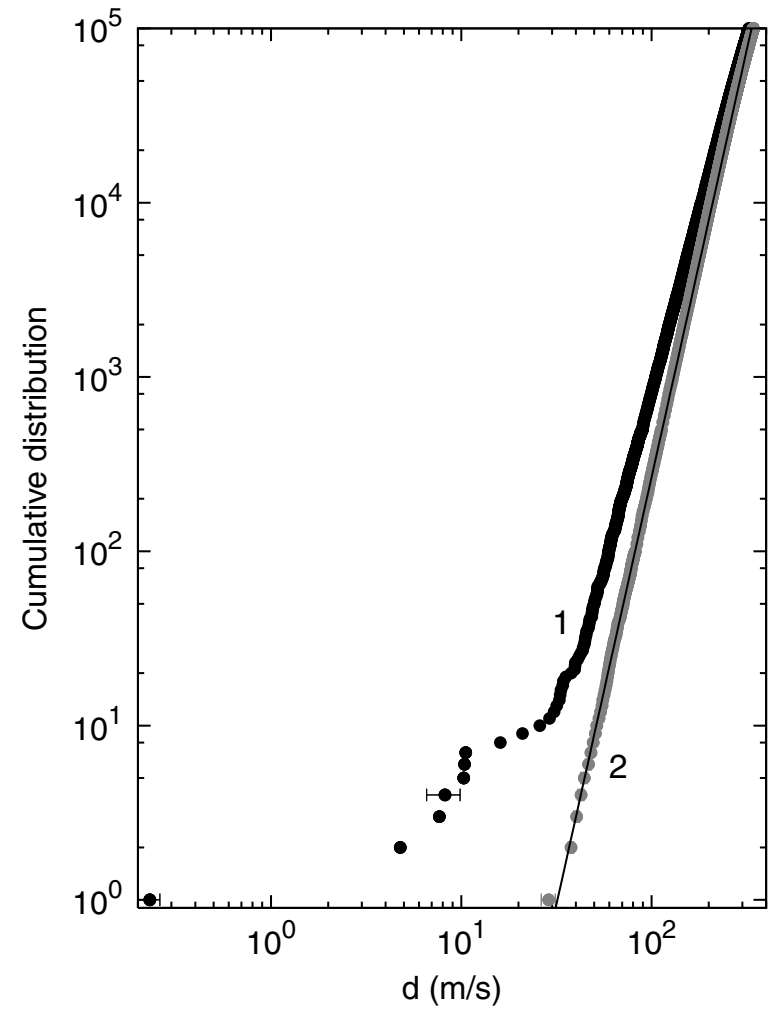

Figure 2. The same as Figure 1 but for the population of $\sim 6250$ Hungaria asteroids. The number of Hungaria pairs with $d<20 \mathrm{~m} \mathrm{~s}^{-1}$ shows an excess over the one expected from a distribution of randomly selected orbits.

asteroid breakups that we know of can explain only a small fraction of the identified pairs. The remaining 45 pairs with $d<10 \mathrm{~m} \mathrm{~s}^{-1}$ may therefore be remnants of the yet-to-becharacterized asteroid collisions or have an entirely different origin.

The orbital distribution of identified pairs across the main belt and Hungaria regions is shown in Figure 3. By analyzing this distribution in detail, we found that 17 identified pairs with $d<10 \mathrm{~m} \mathrm{~s}^{-1}$ are members of prominent asteroid families (e.g., the Vesta family has five pairs; the Flora, Massalia, Gefion, and Eos families have two pairs each). Finally, 22 main-belt pairs with $d<10 \mathrm{~m} \mathrm{~s}^{-1}$ are not members of any known family. The ratio of the family pairs to background pairs thus roughly respects the family-to-background ratio of known main-belt asteroids $(\approx 2: 3$; Nesvorný et al. 2005). This shows that the identified pairs have origins probably unrelated to prominent asteroid families.

The pairs appear to be sampling the orbital location of known asteroids with a preference for small values of $a$. For example, we found four pairs with $d<10 \mathrm{~m} \mathrm{~s}^{-1}$ in the Hungaria region. These eight paired asteroids represent $\approx 1.2 \times 10^{-3}$ of the total of known 6250 Hungarias. Similarly, the fractions of paired asteroids in the inner $(2.0<a<2.5 \mathrm{AU})$, central $(2.5<a<2.82 \mathrm{AU})$, and outer parts $(2.82<a<3.3 \mathrm{AU})$ of the main belt are $\approx 4.4 \times 10^{-4}, \approx 1.1 \times 10^{-4}$, and $\approx 1.2 \times$ $10^{-4}$, respectively. This progression of the pair fraction with $a$ is probably due to the generally small sizes of paired asteroids (see below) and because small (and faint) asteroids represent a larger/smaller fraction of the known population with smaller/larger values of $a$. Alternatively, the progression of pair fraction with $a$ may be a signature of the physical process that produced these pairs. We will discuss these issues in Section 6. 
Table 1

Osculating Orbital Elements of Selected Asteroid Pairs

\begin{tabular}{|c|c|c|c|c|c|c|c|c|}
\hline & Asteroid & $\begin{array}{c}d \\
\left(\mathrm{~m} \mathrm{~s}^{-1}\right)\end{array}$ & $\begin{array}{c}a \\
(\mathrm{AU})\end{array}$ & $e$ & $\begin{array}{c}i \\
(\mathrm{deg}) \\
\end{array}$ & $\begin{array}{c}\Omega \\
(\mathrm{deg})\end{array}$ & $\begin{array}{c}\omega \\
(\mathrm{deg})\end{array}$ & $\begin{array}{c}M \\
(\mathrm{deg})\end{array}$ \\
\hline \multirow[t]{2}{*}{63440} & 2001 MD30 & 0.23 & 1.93809628 & 0.0885952 & 19.98645 & 229.53463 & 205.5398 & 53.0882 \\
\hline & 2004 TV14 & & 1.93809783 & 0.0885978 & 19.98632 & 229.53426 & 205.5597 & 49.8702 \\
\hline \multirow[t]{2}{*}{1270} & Datura & 0.89 & 2.23487789 & 0.2076102 & 5.98966 & 97.88547 & 258.8381 & 110.8520 \\
\hline & 2003 SQ168 & & 2.23483664 & 0.2076011 & 5.99083 & 97.47114 & 259.3936 & 112.4630 \\
\hline \multirow[t]{2}{*}{21436} & Chaoyichi & 1.25 & 2.18645378 & 0.0845606 & 3.73571 & 320.44395 & 177.8815 & 62.5725 \\
\hline & 2003 YK39 & & 2.18650915 & 0.0845216 & 3.73553 & 320.44562 & 177.9246 & 70.7519 \\
\hline \multirow[t]{2}{*}{76111} & 2000 DK106 & 1.49 & 2.71663603 & 0.0482280 & 6.92518 & 50.26390 & 44.2155 & 344.0111 \\
\hline & 2005 JY103 & & 2.71671333 & 0.0481890 & 6.92307 & 50.13812 & 44.4385 & 346.6514 \\
\hline \multirow[t]{2}{*}{5026} & Martes & 1.62 & 2.37726416 & 0.2422318 & 4.29094 & 304.86751 & 17.0708 & 215.0044 \\
\hline & 2005 WW113 & & 2.37713227 & 0.2422590 & 4.28933 & 305.02257 & 17.0068 & 294.0991 \\
\hline 32957 & 1996 HX20 & 2.15 & 2.27788034 & 0.1158256 & 5.92724 & 207.03035 & 177.2563 & 356.0667 \\
\hline \multirow[t]{5}{*}{38707} & 2000 QK89 & & 2.27808240 & 0.1158210 & 5.92559 & 207.26536 & 177.2623 & 23.0195 \\
\hline & 2005 SU152 & 2.45 & 2.64035888 & 0.3183173 & 12.48825 & 54.48537 & 296.9168 & 218.5736 \\
\hline & 2005 UY97 & & 2.64007789 & 0.3182901 & 12.48629 & 54.48376 & 296.9309 & 217.4800 \\
\hline & 2003 YR67 & 2.98 & 2.23627647 & 0.1156745 & 3.84354 & 87.21154 & 196.9591 & 228.4327 \\
\hline & 2005 KB6 & & 2.23604590 & 0.1157418 & 3.84355 & 87.20837 & 196.9388 & 253.2172 \\
\hline \multirow[t]{2}{*}{143155} & 2002 XS50 & 3.20 & 2.86758283 & 0.0577354 & 1.27936 & 215.37411 & 205.8427 & 36.5412 \\
\hline & 2007 TG383 & & 2.86770444 & 0.0576247 & 1.28257 & 215.36125 & 205.7755 & 6.5704 \\
\hline \multirow[t]{4}{*}{17198} & Gorjup & 4.44 & 2.27964308 & 0.1025544 & 3.28775 & 12.31579 & 251.8707 & 339.2613 \\
\hline & 2004 FC126 & & 2.27960032 & 0.1024093 & 3.29136 & 12.54755 & 251.4992 & 340.4865 \\
\hline & 2002 PU155 & 4.69 & 2.29585084 & 0.1782413 & 3.33700 & 105.00972 & 295.0929 & 145.5373 \\
\hline & 2006 UT69 & $\ldots$ & 2.29583828 & 0.1784030 & 3.33433 & 105.27419 & 294.6035 & 126.8154 \\
\hline 6070 & Rheinland & 5.79 & 2.38690224 & 0.2109729 & 3.13222 & 84.01751 & 292.7427 & 188.25826 \\
\hline 54827 & 2001 NQ8 & $\ldots$ & 2.38733830 & 0.2111273 & 3.13115 & 84.02604 & 292.5794 & 223.96543 \\
\hline
\end{tabular}

Notes. Osculating orbital elements are given for epoch MJD 54500. The bold digits indicate the current uncertainty of orbits (i.e., the first uncertain digit at the $1 \sigma$ level). Asteroids 2005 SU152 and 2005 UY97 have the largest orbital uncertainty because they have been observed during a single opposition. The third column gives the distance, $d$, of paired orbits according to Equation (1).

Table 2

The Designations of Asteroids in 60 Identified Pairs with $d<10 \mathrm{~m} \mathrm{~s}^{-1}$

\begin{tabular}{|c|c|c|c|c|c|c|c|c|}
\hline \multirow[b]{2}{*}{$\mathrm{H}$} & \multicolumn{2}{|c|}{ Pairs (a) } & \multicolumn{3}{|c|}{ Pairs (b) } & & \multicolumn{2}{|c|}{ Pairs (c) } \\
\hline & 2001 MD30 & 2004 TV14 & & Tensho-kan & 2003 SF334 & & $2000 \mathrm{WZ} 112$ & 2000 AH207 \\
\hline \multirow[t]{5}{*}{ D } & Datura & 2003 SQ168 & & 2005 UV124 & 2007 RH92 & $\mathrm{D}$ & Datura & 2003 CL5 \\
\hline & Chaoyichi & 2003 YK39 & & 2003 UU192 & 2005 UL291 & & 2000 SP31 & 2007 TN127 \\
\hline & 2000 DK106 & $2005 \mathrm{JY} 103$ & & 1999 TL103 & 2007 TC334 & & 2001 YA114 & 2002 AY48 \\
\hline & Martes & 2005 WW113 & & 2003 QX79 & 2004 RA5 & $\mathrm{D}$ & 2003 CL5 & 2003 SQ168 \\
\hline & 1996 HX20 & 2000 QK89 & $\mathrm{K}$ & 1998 SC49 & 2002 SQ20 & $\mathrm{V}$ & 2002 WZ5 & 2001 UY21 \\
\hline \multirow[t]{2}{*}{ I } & 2005 SU152 & 2005 UY97 & & 2000 SS286 & 2002 AT49 & & 1999 VJ178 & 2001 XH69 \\
\hline & 2003 YR67 & 2005 KB6 & $\mathrm{H}$ & 1999 RP29 & 2001 BV47 & & 2000 ED69 & 2003 WZ36 \\
\hline \multirow[t]{4}{*}{ K } & 2002 XS50 & 2007 TG383 & & 1998 QU12 & 2001 HU24 & & 2006 RA16 & 2007 TD201 \\
\hline & Gorjup & 2004 FC126 & & 1997 UR17 & 2001 XN74 & I & 1999 RV84 & 2003 SA127 \\
\hline & 2002 PU155 & 2006 UT69 & $\mathrm{H}$ & Wasserburg & 2001 XO105 & A & 2002 JH41 & 2002 JZ80 \\
\hline & Toepperwein & 2006 AL54 & $\mathrm{D}$ & 2001 WY35 & 2003 SQ168 & & 1998 RB75 & 2003 SC7 \\
\hline \multirow[t]{3}{*}{$\mathrm{H}$} & $2001 \mathrm{HJ} 7$ & 1999 VA117 & & 2005 LE5 & 2002 RQ273 & & 2002 RW219 & 2002 VW59 \\
\hline & 2005 QV114 & 2007 OS5 & & 2004 TD93 & 2006 BK172 & & 2000 BE34 & 2005 QH8 \\
\hline & 2001 UU227 & 2005 ED114 & & 2003 WA112 & 2003 UU192 & & Moore-Sitterly & 1999 RP27 \\
\hline \multirow[t]{5}{*}{ K } & Pepawlowski & 2003 SB65 & & 2001 HZ32 & 1999 TE221 & K & 1996 AJ7 & 2000 HC49 \\
\hline & 2001 ET15 & 2006 KM53 & & 2000 QV27 & 2002 AL46 & $\mathrm{K}$ & 2001 XL94 & 2005 WV8 \\
\hline & 2000 AJ227 & 2002 TF272 & & 2001 OY21 & 2006 EY16 & $\mathrm{L}$ & Lucascavin & 2003 VM9 \\
\hline & Rheinland & 2001 NQ8 & & 2002 EN153 & 2002 RG72 & $\mathrm{D}$ & 1999 UZ6 & 2003 SQ168 \\
\hline & Linnaea & 1999 RH118 & $\mathrm{D}$ & Datura & 2001 WY35 & & 2000 QY211 & 2004 TJ325 \\
\hline
\end{tabular}

Notes. From top to bottom, the pairs listed in column (a) have $d=0.23 \mathrm{~m} \mathrm{~s}^{-1}$ (2001 MD30 and 2004 TV14) to $d=5.81 \mathrm{~m} \mathrm{~s}^{-1}$ (Linnaea and 1999 RH118). Pairs (b) have $d=6.06-8.66 \mathrm{~m} \mathrm{~s}^{-1}$ and pairs (c) have $d=8.77-$ $10.03 \mathrm{~m} \mathrm{~s}^{-1}$. Labels A, D, I, K, L, and V preceding the first asteroid in the pair denote members of Aeolia, Datura, Iannini, Karin, Lucascavin, and Veritas families. Label $\mathrm{H}$ denotes Hungaria asteroids.

Figure 4(a) shows the distribution of absolute magnitudes, $H$, of asteroids in pairs with $d<10 \mathrm{~m} \mathrm{~s}^{-1}$. The brightest paired ob- ject is (1270) Datura with $H=12.5$ and diameter $D \approx 10.8 \mathrm{~km}$ for estimated albedo $A=0.15$ (Nesvorný et al. 2006a). The 


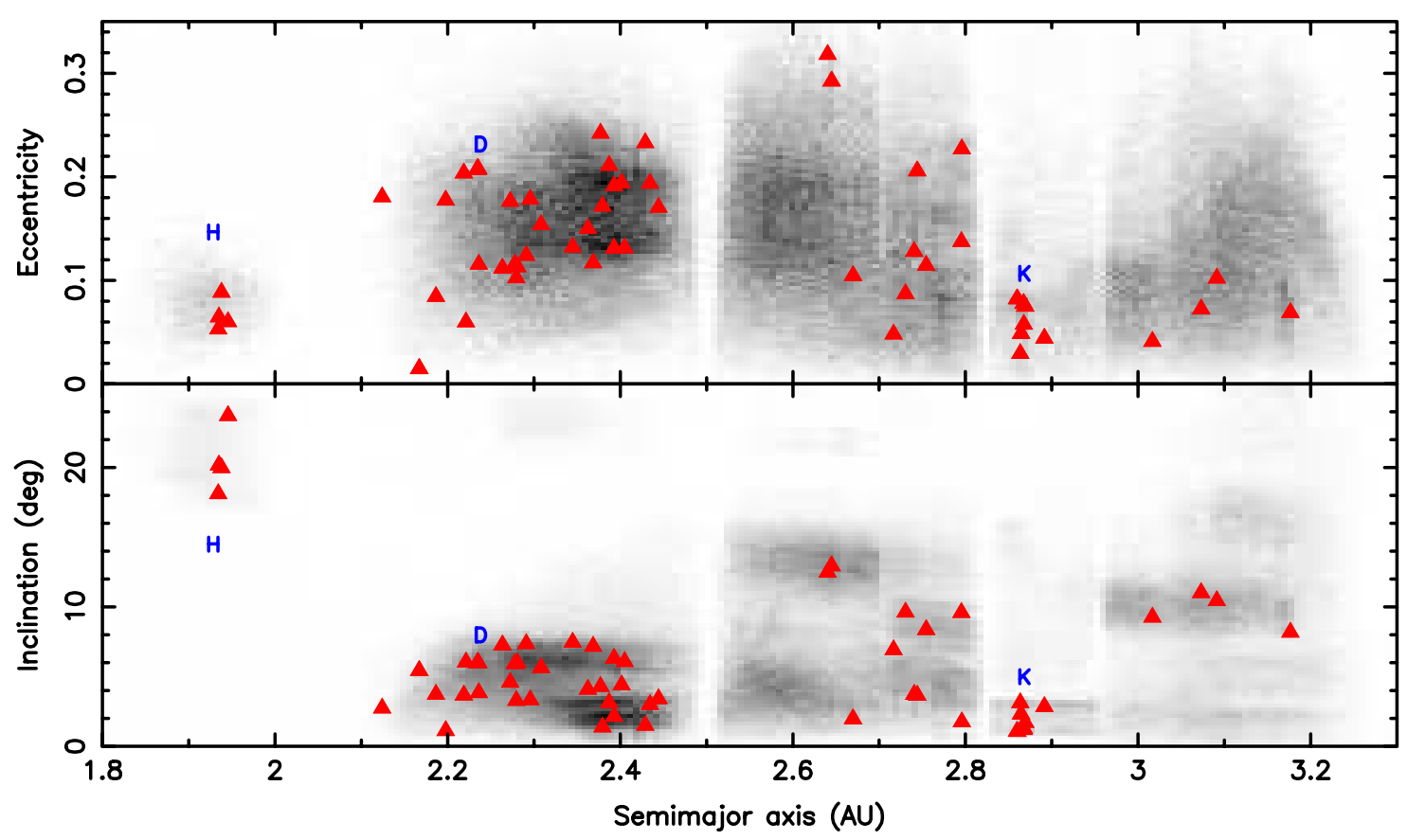

Figure 3. The orbits of 60 identified asteroid pairs with $d<10 \mathrm{~m} \mathrm{~s}^{-1}$ (red triangles). The gray-scale background shows the number density of known asteroids in $(a, e)$ (top panel) and $(a, i)$ (bottom) projections. Letters "H," "D," and "K" denote the orbit locations of Hungaria, Datura, and Karin asteroids; 5, 4, and 5 pairs were found in these groups, respectively.
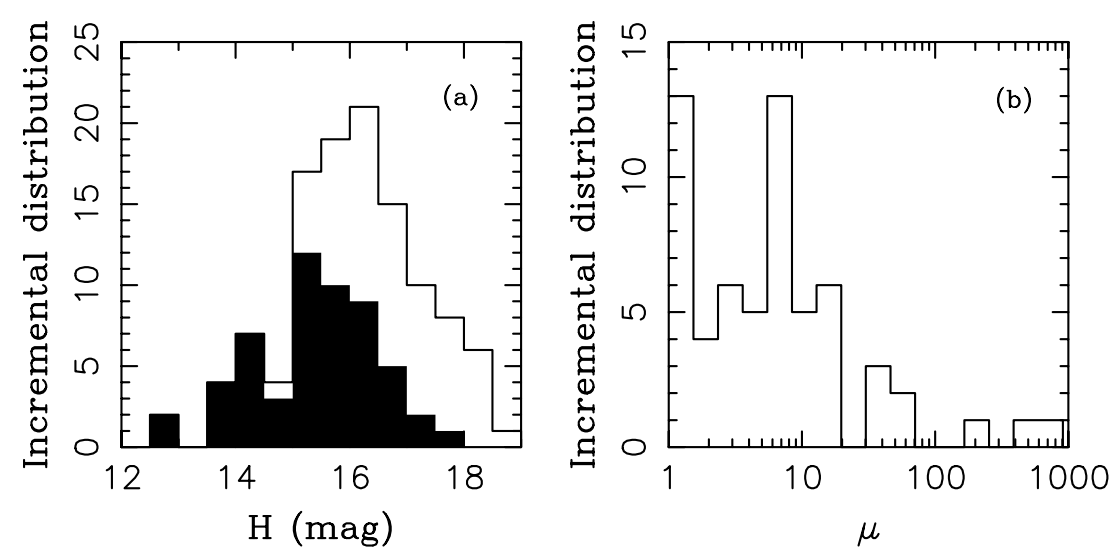

Figure 4. The distribution of absolute magnitude values, $H$, for the 60 identified asteroid pairs with $d<10 \mathrm{~m} \mathrm{~s}^{-1}$ (open histogram in the left panel). The filled histogram shows the distribution of $H$ for the larger objects in each pair. The distribution of the estimated mass ratio, $\mu=m_{1} / m_{2} \sim 10^{0.6\left(H_{2}-H_{1}\right)}$, of the two components in pairs is shown in the right panel. Most pairs have $\mu<20$ with the median value of $\sim 5$.

size distribution of paired objects increases from $H=13$ to $H=15$, has a maximum for $H \approx 15-16.5$ (corresponding to $D \approx 1.7-3.4 \mathrm{~km}$ for $A=0.15$ ), and decreases beyond $H=16.5$ due to the observational incompleteness. The smallest asteroids in the known pairs have sub-km diameters.

Panel (b) in Figure 4 shows the distribution of $\mu=m_{1} / m_{2}$, where $m_{1}$ and $m_{2}$ denote the masses of the larger and smaller objects in each pair, respectively. We determined $\mu$ assuming that the two objects in each pair have the same albedo. Accordingly, $\mu=10^{0.6\left(H_{2}-H_{1}\right)}$, where $H_{1}$ and $H_{2}$ are the absolute magnitudes of the large and small objects in each pair. We found that most pairs have $\mu=1-20$ and only $<10 \%$ pairs have $\mu>100$. The median value of $\mu$ is $\approx 5$. Interestingly, these low $\mu$ values are similar to those of near-Earth asteroid (NEA) binaries and small main-belt asteroid binaries (e.g., Merline et al. 2002; Pravec \& Harris 2007). This may indicate that the physical process producing the asteroid pairs may be similar to that of the NEA binaries. Conversely, the large main-belt binaries with wide separations and the two largest fragments in asteroid families typically have $\mu \gg 10$.

\section{STATISTICAL ANALYSIS}

The orbits of objects in each identified pair with $d<10 \mathrm{~m}$ $\mathrm{s}^{-1}$ are very similar (Table 1 ). Here we show that they cannot be produced by random fluctuations of the asteroid orbit density in 5D space $(a, e, i, \Omega, \varpi)$. We used two methods to estimate the probability that a selected identified pair with distance $d$ occurs as a random fluctuation.

Method 1. In the first method, we randomly distributed 370,000 orbits in 5D orbital element space. The range and number density of these orbits were set to correspond to the size of the asteroid belt in $(a, e, i)$ and the variation of the number density of real asteroids with these elements (e.g., due to 
resonances and prominent asteroid families). Using the method described in Section 2, we then searched for tight pairs in the random distribution of orbits. Finally, we averaged the number of identified pairs with distance $d$ over different realizations of the orbit distribution produced with different seeds of the random generator.

The resulting cumulative distribution, $N(<d)$, is shown in Figure 1 (gray symbols labeled 2). As expected, $N(<d) \propto d^{5}$. For low values of $d$, a gap opens between this distribution and the distribution of real asteroid pairs. For example, based on our statistical test we would expect to have only one pair with $d \approx 10 \mathrm{~m} \mathrm{~s}^{-1}$ if the distribution is random. Instead, there are 60 pairs with $d<10 \mathrm{~m} \mathrm{~s}^{-1}$ among real asteroids. This suggests that the likelihood that one selected real pair occurs due to chance is $\sim 1.7 \%$. The likelihood significantly drops with decreasing $d$; e.g., it is $\approx 0.2 \%$ for $d<4.5 \mathrm{~m} \mathrm{~s}^{-1}$. Figure 2 shows our results for the Hungaria asteroids. In this case, there are nine real pairs with $d \leqslant 20 \mathrm{~m} \mathrm{~s}^{-1}$, each having only $\lesssim 1 \%$ probability to occur by chance.

Method 2. In the second method we draw a box around a pair in $(a, e, i, \varpi, \Omega)$ with volume $V=d^{5}$, where $d$ corresponds to the separation distance of the paired orbits. Assuming that the local number density of orbits is $\eta(a, e, i, \varpi, \Omega)$, the number of orbits expected to be found in $V$ is $v=\eta V$, where $v$ is typically some small number. The probability of finding $n$ orbits in $V$ is given by the Poisson statistics

$$
p_{n}(d)=\frac{v^{n}}{n !} e^{-v}
$$

where the special case with $n=2$ interests us most here. The probability of finding $n$ orbits in any box of volume $V$ then is

$$
P_{n}(d)=\sum_{M} p_{n}(d)=\frac{V^{n-1}}{n !} \int d V \eta^{n} e^{-v},
$$

where we substituted the sum over all $M$ boxes with volume $V$ by the integral over 5D space. Because $e^{v} \approx 1$ for small $v$, the above expression could be further simplified yielding

$$
P_{n}(d) \approx \frac{1}{n !} \frac{\left\langle\eta^{n}\right\rangle V_{\mathrm{tot}}^{n}}{M^{n-1}}
$$

where $\left\langle\eta^{n}\right\rangle$ is the mean $\eta^{n}$ of the main-belt asteroids in 5D space, $V_{\text {tot }}$ is the total 5D volume of the asteroid belt, and $M=V_{\text {tot }} / V$.

In a special case with constant $\eta$, Equation (4) can be written as

$$
P_{n}(d) \approx \frac{1}{n !} \frac{N^{n}}{M^{n-1}},
$$

where $N \approx 370,000$ is the total number of orbits in our case. Note that this last equation is the same as Equation (A1) of Nesvorný \& Vokrouhlický (2006) for $n=3$ and in the limit of large $N$. Therefore, for constant $\eta$ and $n=2$,

$$
P_{2}(d) \approx \frac{1}{2} \frac{N^{2}}{M}
$$

In order to be more realistic, we used Equation (4) to determine $P_{2}(d)$ where $\eta$ is not constant. To calculate $\left\langle\eta^{2}\right\rangle$, we accounted for the variation of asteroid orbit density across the main belt produced by known asteroid families. Specifically, we assumed that $\eta \neq f(\varpi, \Omega)$, because orbits in large asteroid families have nearly uniform distribution of $\varpi$ and
$\Omega$, and determined $\eta=\eta(a, e, i)$ numerically by smoothing the asteroid orbit density over a desired distance, $d_{\text {smooth }}$. In practice, the smoothing distance can be characterized by $\Delta a=d_{\text {smooth }} /\left(n \sqrt{k_{a}}\right)$, where we used Equation (1) to link $d_{\text {smooth }}$ to a semimajor axis interval, $\Delta a$.

With $d=5 \mathrm{~m} \mathrm{~s}^{-1}$ and constant $\eta$, we find that $M \approx 10^{13}$. From Equation (6), we have that $P_{2}(5) \approx 2 \times 10^{-3}$ (under a constant number density assumption). This would indicate that the probability of having one pair due to random fluctuation is negligible. The probability increases, however, if varying $\eta$ is taken into account in Equation $(4)$. We obtained $P_{2}(5) \approx 0.01$ for $\Delta a=0.1 \mathrm{AU}$ and $P_{2}(5) \approx 0.03$ for $\Delta a=0.01 \mathrm{AU}$. Therefore, the probability increases by a factor of 3 if the resolution is increased by a factor of 10 . This shows that the most probable locations of tight asteroid pairs produced by random fluctuations should be found in tight asteroid families where the number density is the highest. We will address this issue below. Still, even with $\Delta a=0.01 \mathrm{AU}$, there is only $\approx 3 \%$ likelihood to find one pair with $d=5 \mathrm{~m} \mathrm{~s}^{-1}$ in the main belt due to random fluctuations.

Young asteroid families. To look into the effect of small-scale fluctuation of the asteroid density in more detail, we focus on the region of the Karin cluster at $a \approx 2.865 \mathrm{AU}$. This family formed $\approx 5.75$ Myr ago by a collisional breakup of an $\approx 40 \mathrm{~km}$ asteroid (Nesvorný et al. 2002, 2006b; Nesvorný \& Bottke 2004). The osculating element range of this family is $a=2.861-2.871 \mathrm{AU}$, $e<0.08$, and $i=0.8^{\circ}-3.3^{\circ}$. As mentioned in Section 2, five pairs with $d<10 \mathrm{~m} \mathrm{~s}^{-1}$ were identified in the Karin cluster. The tightest of these pairs is the pair of asteroids (143155) 2002 XS50 and 2007 TG383 with $d=3.2 \mathrm{~m} \mathrm{~s}^{-1}$. To estimate that this pair occurs due to chance fluctuation, we applied Equation (4) where $\eta=\eta(a, e, i)$ was smoothed with $\Delta a=0.0001$ AU. We found that the probability to have one pair with $d=3.2 \mathrm{~m} \mathrm{~s}^{-1}$ in the Karin cluster region would be only $\approx 1 \%$. This would indicate that the identified pairs need some special explanation.

By analyzing this case in more detail, we found that $(\varpi, \Omega)$ are correlated in complicated ways with $(a, e, i)$. This may be understood from the recent formation of the Karin cluster. Initially, soon after their ejection from the collision site, the Karin family fragments were launched into space with correlated $a$ and $e$, and a very tight dispersion in $\varpi$ and $\Omega$. Moreover, hydrodynamic simulations show that the orbit distribution of the ejected fragments has a complicated structure with voids and overdense regions located along preferred directions (Nesvorný et al. 2006b). These structures are not completely erased over $5.75 \mathrm{Myr}$ of orbit evolution. Therefore, the distribution of $(a, e, i, \varpi, \Omega)$ in the recently formed families is fractal and more apt to yield tight pairs than would be expected if $\eta \neq f(\varpi, \Omega)$. We verified this by using Karin's $\eta=\eta(a, e, i, \varpi, \Omega)$ in Equation (4). Probability $P_{2}(3.2)$ becomes of order of unity in these tests. We therefore find that the origin of pair (143155) 2002 XS50 and 2007 TG383, and other pairs identified in the recently formed families, probably does not require any special explanation. These are pairs of fragments launched by different impacts onto very similar orbits.

Conversely, our probability estimates suggest that the remaining $\sim 45$ identified pairs outside young asteroid families and with $d<10 \mathrm{~m} \mathrm{~s}^{-1}$ need a special explanation. These objects are located in the Hungaria region (4 objects), old asteroid families (17), and main-belt background population (22). We conclude that some, yet-to-be-identified physical process may be affecting asteroids, producing majority of the observed pairs. We will discuss various possibilities in Section 6. 


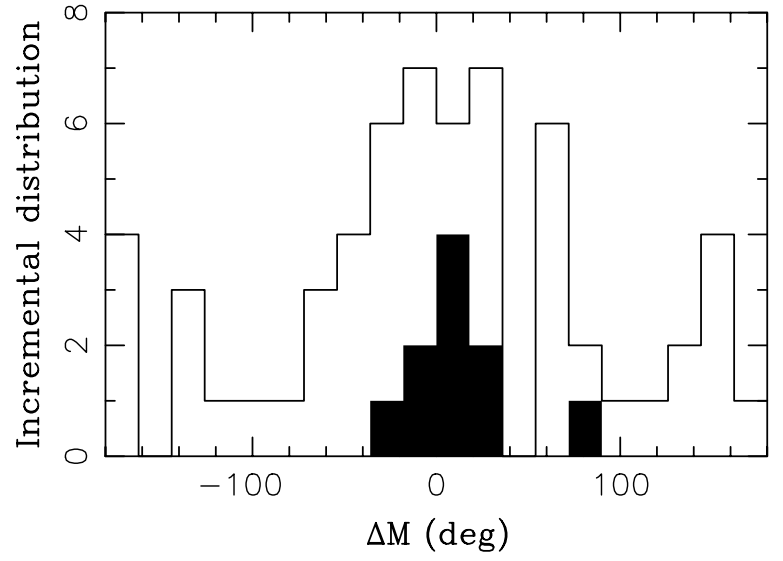

Figure 5. The distribution of differential mean anomaly values, $\Delta M$, for 60 identified pairs with $d<10 \mathrm{~m} \mathrm{~s}^{-1}$ (open histogram). The distribution of $\Delta M$ for pairs with $d<4.5 \mathrm{~m} \mathrm{~s}^{-1}$ (Table 1) is shown by the filled histogram. The latter distribution is clearly peaked near $\Delta M=0^{\circ}$.

\section{FORMATION TIME ESTIMATES}

To get insights into the nature of the physical process that could have produced the identified pairs, we attempt here to estimate when the pairs formed. We first noted that the orbits of paired asteroids typically have a very small difference of osculating $\Omega$ (and $\varpi$ ) (see Table 1). This shows that these angles have not been modified much by planetary perturbations. By testing the rate of spreading of $\Omega$ (and $\varpi$ ) over time, we found that most pairs must have formed $\lesssim 1$ Myr ago because $\Omega$ (and $\varpi$ ) of paired orbits diverge over longer timescales. Additionally, differences in mean anomaly, $\Delta M$, of individual cluster members with $d<10 \mathrm{~m} \mathrm{~s}^{-1}$ are currently distributed approximately evenly between $0^{\circ}$ and $360^{\circ}$ showing that the Keplerian shear had enough time to operate (Figure 5). Given these results, we estimate that the age of pairs, $t_{\text {age }}$, cannot generally be much younger than $\sim 10 \mathrm{kyr}$ and older than $\sim 1 \mathrm{Myr}$.

We note, however, that our ten tightest pairs with $d<$ $4.5 \mathrm{~m} \mathrm{~s}^{-1}$ have their $\Delta M$ values clustered near $0^{\circ}$ (Table 1 and Figure 5). Either these pairs are only several thousand years old $^{4}$ or, and perhaps more likely, they happen to have small $d$, because $\Delta M \sim 0^{\circ}$ at the current epoch implies that the shortperiod variations of paired orbits are "in phase." We tested the latter possibility by tracking the orbit evolution of different pairs into the past and future epochs. We found that the smallest $d$ values for a given pair indeed occur for $\Delta M \sim 0^{\circ}$. Figure 6 shows result from a numerical experiment where we launched a fragment from asteroid (1270) Datura with a relative speed $0.5 \mathrm{~m} \mathrm{~s}^{-1}$. The orbits of these two bodies were tracked into the future for $500 \mathrm{kyr}$. We note that epochs of $\Delta M \sim 0^{\circ}$ are strongly correlated with those of $\Delta a \sim 0$ AU. As a result, these moments also define the location of deep minima in $d$ (top panel). The

4 Assuming that the Keplerian shear produced $\Delta M$ of a selected pair, the age of the pair, $t_{\text {age }}$, can be estimated as

$$
t_{\text {age }} \sim \frac{|\Delta M|}{3 \pi} \frac{a}{\Delta a} P_{\text {orb }},
$$

where $a$ is the semimajor axis, $P_{\text {orb }}$ is the orbital period, and $\Delta a$ is the mean semimajor axis separation of the two objects in the pair. This latter value cannot be taken from the current difference of the osculating semimajor axis values (e.g., $\sim 4 \times 10^{-6} \mathrm{AU}$ for the tightest pair of asteroids (63440) 2001 MD30 and 2004 TV14). Instead, it is set by the short-period variations of the asteroid's semimajor axis due to planetary perturbations. Typically, $\Delta a \sim 2 \times 10^{-3} \mathrm{AU}$ (see, e.g., Figure 6) age of the pair may not necessarily be very small, but rather punctuated by near-entire synodic periods of the relative motion in the pair. This suggests that the two objects in each pair with $d<4.5 \mathrm{~m} \mathrm{~s}^{-1}$ are probably not very different from those with $4.7<d<10 \mathrm{~m} \mathrm{~s}^{-1}$ except they have, by chance, small $\Delta M$ at the current epoch.

Working under the assumption that the two asteroids in a pair were once part of the same object, a more precise estimate of the time when they separated from each other can be obtained by tracking their position vectors backward in time and showing that they converge. Ideally, since the objects in the identified pairs are typically $\sim 1$ to a few $\mathrm{km}$ across, we would need to show that their positions converge to within a few $\mathrm{km}$. This is unfortunately unrealistic because it is difficult to track the location of orbiting objects with such a precision over the required timescales. In several cases described in Section 5, however, we were at least able to demonstrate a possible recent encounter of the two bodies in a pair to within $1000 \mathrm{~km}$. According to our tests (described below), such a deep and recent encounter obtained with our backward-tracking method can be a signature of the pair's recent formation event.

We numerically integrated the orbits of all pairs with $d<$ $10 \mathrm{~m} \mathrm{~s}^{-1}$ backward in time with the code known as SWIFT_MVs (Levison \& Duncan 1994) and 3.65 day time step. The initial orbits and tracking method were set to account for three important factors: (i) the osculating orbits of asteroids are known with imperfect accuracy, (ii) the thermal Yarkovsky force that can change the semimajor axis of small asteroids (e.g., Bottke et al. 2006), and (iii) the effects of chaos produced by planetary gravitational perturbations. Effect (ii) is especially important because the slow drift of orbits in $a$ due to the Yarkovsky effect can produce amplified effects on other orbital elements (e.g., Vokrouhlický et al. 2000).

To deal with (i), we cloned the orbit of each asteroid assuming the normal distribution of orbit nonsingular, equinoctical elements, and $1 \sigma$ uncertainties that we calculated for each individual object using the ORBFIT9 public software (http://newton.dm.unipi.it/orbfit/). In total, 20 orbit clones were numerically integrated for each asteroid. In addition, to cope with (ii), we used 51 "yarko" clones for each of the two paired orbits that were assigned different values of $d a / d t$ (secular value of the semimajor axis drift rate). The range of these values was determined from the linearized theory of the diurnal Yarkovsky effect (e.g., Vokrouhlický 1999). For that purpose, we converted the observationally determined absolute magnitudes of the asteroids to their diameters using a geometric albedo value $p_{V} \sim 0.3$ for the main belt and $p_{V} \sim 0.4$ for the Hungaria objects. With that we conservatively overestimate maximum $d a / d t$ values. In order to simplify our work, we replaced the full formulation of the Yarkovsky force with alongtrack acceleration $\frac{1}{2} n(n a / v)(d a / d t)$, with $n$ being the orbital mean motion, $a$ the orbital semimajor axis, and $v$ the instantaneous orbital velocity. Such perturbative acceleration produces the same averaged semimajor axis drift $d a / d t$ as expected from the theory of the Yarkovsky effect. With that, we only span the admissible $d a / d t$ value and do not need to sample a much larger parameter space of the Yarkovsky forces. With this approach we cannot reproduce the possible off-plane displacements due to the Yarkovsky forces, but we argue in the next section that they are at most comparable to our numerical method resolution.

In total, we produced 1020 possible past orbit histories for each asteroid that differ by the starting orbit and magnitude of Yarkovsky thermal drag. To determine $t_{\text {age }}$ for a specific 

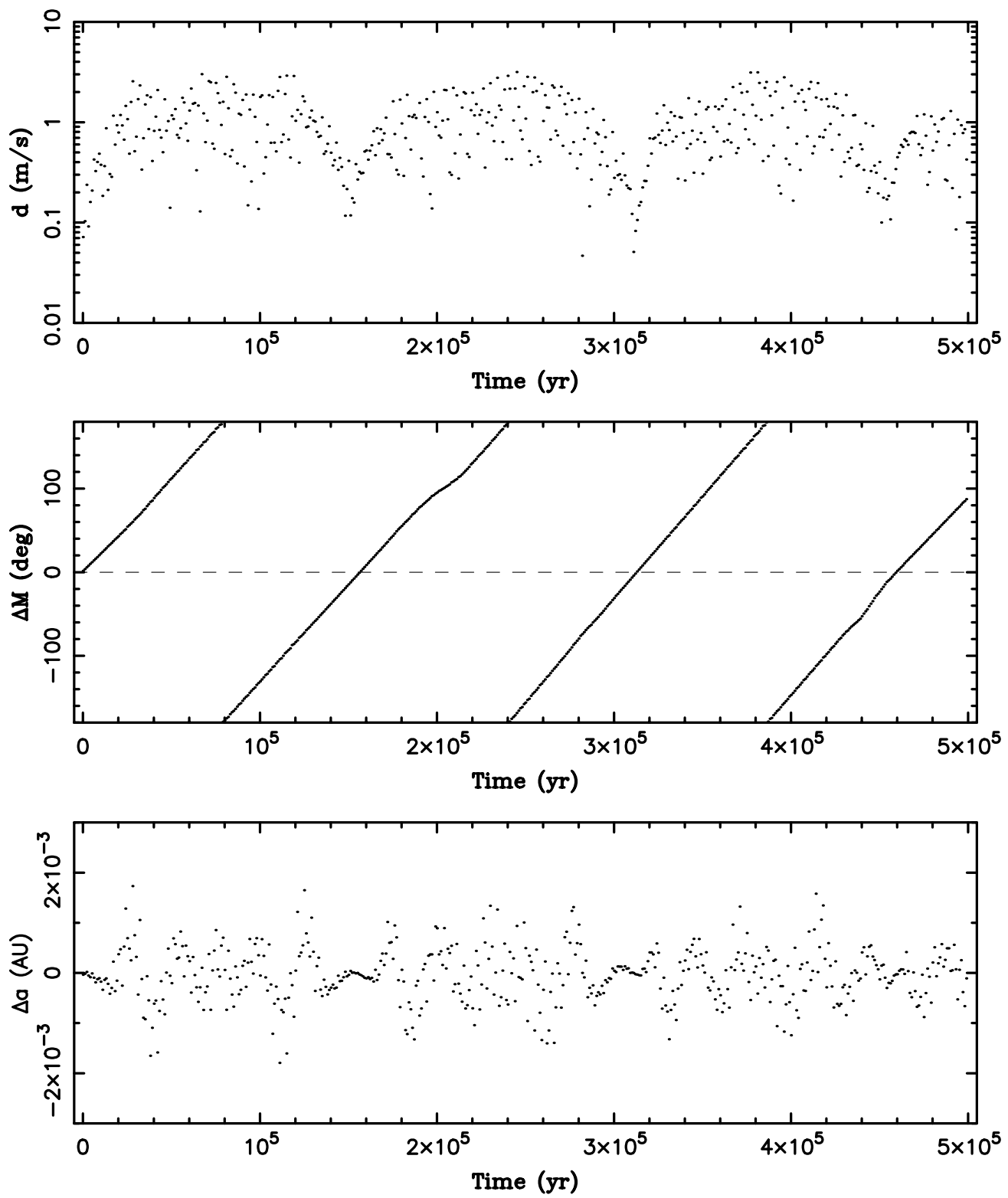

Figure 6. Results of a numerical experiment indicating $\Delta M \sim 0^{\circ}$ in the pairs of asteroids ( $M$ is the mean anomaly) preferentially leads to small $d$ values. A test asteroid was launched from asteroid (1270) Datura with relative speed $0.5 \mathrm{~m} \mathrm{~s}^{-1}$. The orbits of these two bodies were numerically tracked into the future (the Yarkovsky forces were not included here). From top to bottom, the panels show distance $d$ (defined in Equation (1)), difference between the two orbits in the mean anomaly ( $\Delta M$ ), and difference in the semimajor axis $(\Delta a)$. These three parameters are clearly correlated. The smallest values of $d$ occur when $\Delta M \sim 0^{\circ}$. Thus an extremely small value of $d$ does not necessarily imply that the age of the pair, since the bodies separated, is extremely small; it only correlates with $\Delta M \sim 0^{\circ}$, a situation that repeats a number of times in the future.

asteroid pair, we selected time $t$ and one recorded orbital history for each of the two asteroids in the pair. We then determined the physical distance, $\Delta(t)$, between the two asteroids at time $t$ for this trial. The same calculation was repeated over all $\left(1020 \times 1020 \sim 10^{6}\right)$ combinations of distinct orbit histories of asteroids in the pair. Eventually, we selected the trial that leads to the minimal physical distance, $\delta(t)$, of asteroids at $t$ and repeated the procedure over different values of $t$ between present and $3 \times 10^{5}$ yr ago. The $t$ values were spaced by $100 \mathrm{yr}$. The range of plausible $t_{\text {age }}$ values was inferred from the functional dependence of $\delta$ on $t$.

We found that the determined $t_{\text {age }}$ values are generally not unique. Typically, one to a few close encounters between paired objects occur within the past $\sim 50 \mathrm{kyr}$ and a continuous range of the $t_{\text {age }}$ values is found for $t \gtrsim 50 \mathrm{kyr}$. This result has an obvious cause. Close encounters between objects can only occur near conjunctions of the two objects in a pair during their orbital motion around the Sun. Without the Yarkovsky thermal drag, these solutions would happen in regular intervals defined by the difference in orbital periods of the pair objects (i.e., synodic period of their mutual motion) and thus by the difference of their (mean) semimajor axis values. It turns out that for the identified pairs with $d<10 \mathrm{~m} \mathrm{~s}^{-1}$, these conjunctions typically occur each $\sim 10-30 \mathrm{kyr}$ (and only longer if the semimajor axis difference is very small; Figure 6). They produce the $t_{\text {age }}$ values spaced by $\sim 10-30$ kyr that we found for $t \lesssim 50 \mathrm{kyr}$. On longer time intervals, the Yarkovsky effect is capable of producing changes in $a$ that are large enough, for asteroids of a typical size in the pairs, to change the timing of conjunctions and allow them to happen at any $t$. This leads to a continuous range of $t_{\text {age }}$ 
values. ${ }^{5}$ Therefore, in general, $t_{\mathrm{age}}$ cannot be precisely determined for most pairs without additional information about the magnitude of the Yarkovsky effect.

\section{INDIVIDUAL CASES}

We applied the method explained in Section 4 to all 60 pairs with $d<10 \mathrm{~m} \mathrm{~s}^{-1}$ and found a few interesting cases where $t_{\text {age }}$ can be reasonably constrained. The most outstanding of these cases is the pair of asteroids (6070) Rheinland and (54827) 2001 NQ8 (Table 1).

\section{1. (6070) Rheinland and (54827) 2001 NQ8}

The present osculating orbits of these two asteroids are separated in $a, e$, and $i$ by $\approx 10^{-4} \mathrm{AU}, \approx 3 \times 10^{-4}$, and $\approx 10^{-3} \mathrm{deg}$, respectively, and differ by $<0.5^{\circ}$ in angles $\Omega$ and $\varpi$. This pair has $d=5.8 \mathrm{~m} \mathrm{~s}^{-1}$. It is somewhat special among all identified pairs because the two objects are relatively big (diameters $D \approx 4.6$ and $1.8 \mathrm{~km}$ ) and have small orbit uncertainty.

When propagated into the past (6070) Rheinland and (54827) 2001 NQ8 experience a deep encounter at $\approx 17 \mathrm{kyr}$ ago, where $^{6} \delta \approx 250 \mathrm{~km}$ (Figure 7). For a comparison, the Hill sphere radius of (6070) Rheinland is about $900 \mathrm{~km}$. Such a deep encounter is not expected on statistical grounds because the torus occupied by the two orbits is $\gtrsim 10^{5} \mathrm{~km}$ wide. Instead, we believe that (6070) Rheinland and (54827) 2001 NQ8 have separated from their common ancestor object at $\approx 17 \mathrm{kyr}$. We performed several tests to support this conclusion.

As a justification of our method, we created a pair of test objects with the first one having the orbit and size identical to those of (6070) Rheinland. The second test object with the size of (54827) 2001 NQ8 was launched from (6070) Rheinland's present location with speed $0.5 \mathrm{~m} \mathrm{~s}^{-1}$ and directions that were chosen differently in different tests. The orbits of these two test objects were tracked from the current epoch, $t_{0}$, forward in time to $t=t_{0}+\tau$ with the selected $d a / d t$ values. We then produced 1020 orbit and "yarko" clones for each object and backtracked these clones first to $t_{0}$ and then to $t=t_{0}-100 \mathrm{kyr}$ in the past. The method described in Section 4 was blindly applied to these orbit histories to determine $\delta(t)$. We found that we were reliably able to show that $\delta(t)$ has a prominent minimum at $t_{0}$ with $\delta\left(t_{0}\right) \sim 500 \mathrm{~km}$, except if $\tau \gtrsim 100 \mathrm{kyr}$. It was impossible to reliably backtrack the formation event for these large $\tau$. For large $\tau$, we found that $\delta(t) \gtrsim 10^{5} \mathrm{~km}$ for any $t_{0}+\tau>t>t_{0}-$ 100 kyr.

\footnotetext{
5 One easily verifies that a characteristic timescale $t_{\mathrm{yar}}$ to spread the orbit position uncertainty to the whole orbit by the unknown Yarkovsky drift $d a / d t \sim(1-3) \times 10^{-4} \mathrm{AU} \mathrm{Myr}^{-1}$ is$$
t_{\mathrm{yar}} \sim\left[\frac{n a}{3 \pi(d a / d t)}\right]^{1 / 2} P_{\mathrm{orb}},
$$

where $n$ is the mean motion, $a$ is the orbital semimajor axis, and $P_{\text {orb }}$ is the orbital period. We typically obtain $t_{\mathrm{yar}} \sim 50-100 \mathrm{kyr}$.

6 We note that the order of magnitude $\delta z$ of the neglected off-plane

Yarkovsky acceleration $a_{\text {yar }}$ can be estimated by (see, e.g., the Appendix in Vokrouhlický et al. 2005)$$
\delta z \sim \frac{1}{2} \frac{a_{\mathrm{yar}}}{n^{2}} \frac{P_{\Omega}}{P_{\mathrm{orb}}},
$$

where $n$ is the orbital mean motion, $P_{\text {orb }}$ is the orbital period, and $P_{\Omega}$ is the characteristic period of node precession. For km-size asteroids in the main belt, we typically obtain $\delta z \sim 100-500 \mathrm{~km}$ as an upper bound of the neglected off-plane effect. This is comparable with our best-achieved $\delta(t)$ for the (6070) Rheinland-(54827) 2001 NQ8 pair.
}

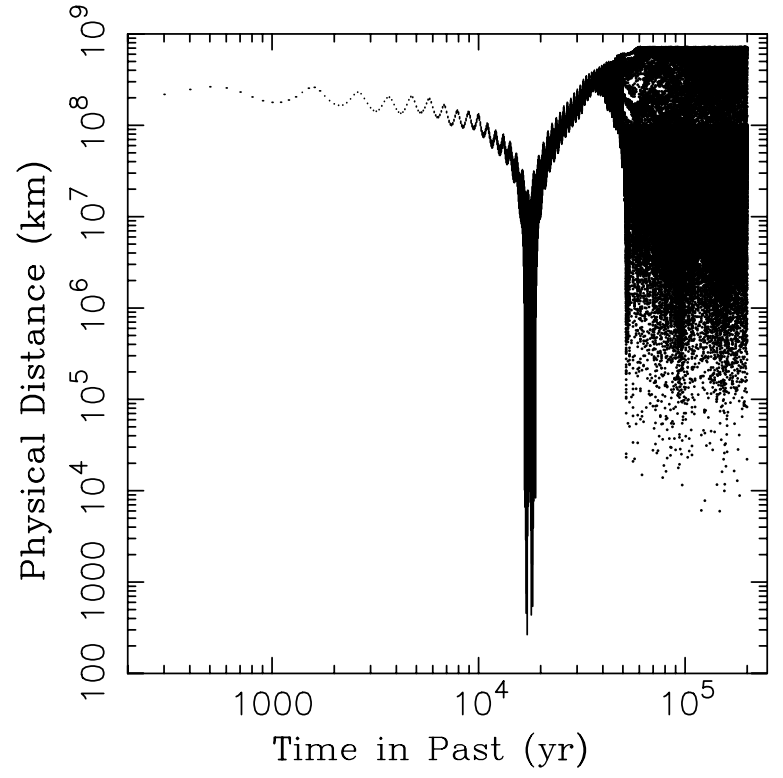

Figure 7. The physical distance $\Delta(t)$ between asteroids (6070) Rheinland and (54827) 2001 NQ8 as a function of time $t$ achieved by comparison of 1020 clone histories for each of the two bodies. We associate the deep encounter, with a minimum modeled physical distance of $\delta_{\min } \approx 250 \mathrm{~km}$, at $t \approx 17 \mathrm{kyr}$ with the formation epoch of this asteroid pair.

Therefore, either the formation event is young $\left(t_{\text {age }} \lesssim 50 \mathrm{kyr}\right)$ and a prominent minimum with $\delta \sim 500 \mathrm{~km}$ is expected or the formation event is old ( $\left.t_{\text {age }}>50 \mathrm{kyr}\right)$ and $\delta \gtrsim 10^{5} \mathrm{~km}$ for any $t$. We mentioned above that the principal cause of this degeneracy at large $t_{\text {age }}$ values is due to the unknown Yarkovsky forces on the two bodies. A minor role is played by the orbital uncertainty and the inherent chaoticity of the asteroids' motion as witnessed by the Lyapunov exponent of $\sim 60 \mathrm{kyr}$. Since our results for the real pair of asteroids (6070) Rheinland and (54827) $2001 \mathrm{NQ8}$ show prominent minimum $\delta \approx 250 \mathrm{~km}$ at $\approx 17 \mathrm{kyr}$, we believe that the age of this pair is about $17 \mathrm{kyr}$. Moreover, by analyzing the formation event of (6070) Rheinland and (54827) 2001 NQ8 with more time resolution, we conclude that $t_{\text {age }}=16.5-19 \mathrm{kyr}$ for this pair.

Interestingly, the relative speed of the two bodies during the encounter at $\approx 17 \mathrm{kyr}$ is only $\approx 0.25 \mathrm{~m} \mathrm{~s}^{-1}$. For a comparison, the ejection speed from a spherical, diameter $D=5 \mathrm{~km}$ object with bulk density $\rho=2.5 \mathrm{~g} \mathrm{~cm}^{-3}$ is $\approx 3 \mathrm{~m} \mathrm{~s}^{-1}$. Therefore, the objects must have separated very gently. Moreover, we found that the component of the separation speed perpendicular to the orbit is typically only $\sim 3 \mathrm{~cm} \mathrm{~s}^{-1}$, while the other two components are almost 1 mag larger. This may suggest that the separation trajectories of the two objects were located within the orbital plane. We discuss the possible implication of this result in Section 6.

\subsection{Results for Other Pairs}

Several identified pairs have $\delta(t)$ similar to that shown in Figure 7. In none of these cases, however, is the minimum value of $\delta$ as low as in the case of pair discussed above. Therefore, we are less confident whether $t_{\text {age }}$ determined by our method corresponds to the pair's actual age. Improved orbit determination and additional information about the strength of the Yarkovsky drag will be needed in these cases to obtain a more reliable result.

For many pairs, we were at least able to place a lower limit on $t_{\text {age }}$. As an example, we discuss the interesting case of the 


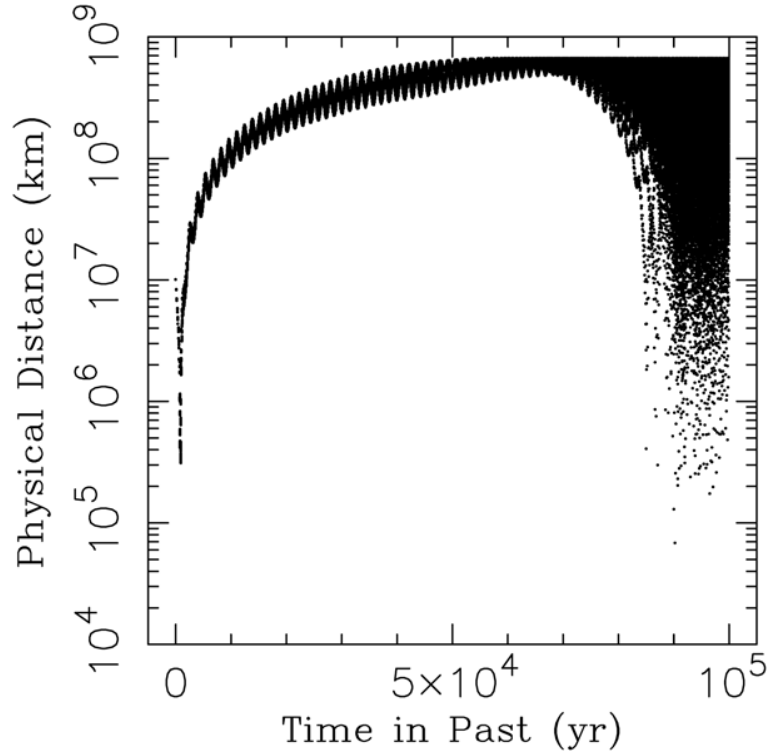

Figure 8. The physical distance $\Delta(t)$ between asteroids (1270) Datura and 2003 SQ168 as a function of time $t$ achieved by comparison of 1020 clone histories for each of the two bodies. The distribution shows only a very distant encounter at $\sim 1 \mathrm{kyr}$ ago and a continuous range of encounters for $t>80 \mathrm{kyr}$. This pair probably formed more than $\sim 100$ kyr ago and probably dates back to the Datura family formation $\approx 450 \mathrm{kyr}$ ago (Nesvorný et al. 2006).

pair of asteroids (1270) Datura and 2003 SQ168. Both these objects are members of the Datura family which formed by a collisional breakup of an inner main-belt asteroid $450 \pm 50 \mathrm{Myr}$ ago (Nesvorný et al. 2006a). Figure 8 shows the $\Delta(t)$ values for this pair. Due to small $\Delta M$ at present, the last conjunction between objects in this pair occurred only $\sim 1000$ yr ago. In this conjunction, $\delta \approx 3 \times 10^{5} \mathrm{~km}$ which indicates a very distant encounter. Apparently, the two asteroids could not have separated during this conjunction. Figure 8 then implies that $t_{\text {age }} \gtrsim 100 \mathrm{kyr}$ and probably several $100 \mathrm{kyr}$ old. This is comparable to the age of the Datura family. We therefore believe that asteroids (1270) Datura and 2003 SQ168 are fragments liberated from their parent object $\approx 450 \mathrm{kyr}$ ago when the Datura family formed. Other pairs with $d<10 \mathrm{~m} \mathrm{~s}^{-1}$ found in young families, 15 in total (e.g., the pair of asteroids 1431552002 XS50 and 2007 TG383 with $d=3.20 \mathrm{~m} \mathrm{~s}^{-1}$ is part of the Karin family), probably also have a collisional origin that dates back to their parent family formation.

We therefore see that the low $\Delta M$ values of tight pairs listed in Table 1 are not necessarily a signature of their extremely young age (also see Section 2). Specifically, the objects in pairs 63440, the tightest of all (Table 1), and 32957 diverge in $M$ and have $t_{\text {age }} \gtrsim 40 \mathrm{kyr}$. The pair 143155 shows $\delta(t)$ behavior similar to the one described for (1270) Datura-2003 SQ168 above. Pairs 5026 and 17198 have $t_{\text {age }} \gtrsim 35 \mathrm{kyr}$ where $\delta(t)$ shows a continuous range of solutions. Both 2003 YR67 and 2002 PU155 show a shallow minimum of $\delta(t)$ for $t \approx 10 \mathrm{kyr}$, but are probably much older than that. Finally, the orbits in pair 2005 SU152 are not known well enough to make our age determination feasible for this pair.

\section{DISCUSSION}

The asteroid pairs identified in this work have an unknown origin. It seems very likely that the paired objects probably represent fragments of disrupted asteroids. The mechanism of the breakup, however, is less certain. Here we discuss various possibilities.

Catastrophic collision. The pairs may have been produced by disruptive collisions. This seems to be especially likely for the 15 pairs with $d<10 \mathrm{~m} \mathrm{~s}^{-1}$ that were found in the young families (e.g., pair (1270) Datura and 2003 SQ168 in the Datura family). These objects were probably ejected in almost identical trajectories producing orbits that stayed very similar until present. Recent hydrodynamic simulations of impacts show that such paired trajectories of fragments can be indeed produced in catastrophic collisions (e.g., Nesvorný et al. 2006b). If the other 45 identified pairs with $d<10 \mathrm{~m} \mathrm{~s}^{-1}$ are parts of yet-to-be-characterized collisional families, we should soon be seeing new objects being discovered with orbits within $d \sim$ $10 \mathrm{~m} \mathrm{~s}^{-1}$ to the paired asteroids using data provided by the new generation sky surveys such as PanSTARRS (e.g., Jedicke et al. 2007).

Several properties of the identified pairs may suggest that at least some of them may have formed by a different physical process than collisions. For example, most pairs have $\mu \leqslant 20$ (see Section 2 and Figure 4(b)) while this mass ratio is typically $\gg 10$ between the largest and other fragments in known asteroid families. Here, however, our inability to detect sub-km main-belt asteroids in small families could have biased the sample toward super-catastrophic collisional breakups that show $\mu \sim 1-10$ (Durda et al. 2007).

The second and perhaps more solid argument against the collisional origin of paired asteroids is their relative abundance in the Hungaria region $\left(\sim 10^{-3}\right.$; see Section 2$)$ versus the inner main-belt region $\left(\sim 2 \times 10^{-4}\right)$. The inner main belt ( $2.1<a<2.5 \mathrm{AU})$ is collisionally coupled to the massive population of asteroids beyond 2.5 AU. Conversely, the orbits of Hungaria asteroids overlap with the inner main belt only, which represents only $\sim 10 \%$ of the total population of mainbelt asteroids. Therefore, we would expect that the collisional activity in the Hungaria region is much lower compared to the inner main belt. Yet, the relative abundance of paired asteroids is $\sim 4$ times higher in the Hungaria region than it is in the inner main belt. (It is not clear, however, how the estimated fractions are effected by the limiting size of asteroids that are observationally detected at different $a$ (see Section 2). We believe that a bias can contribute to the very low fraction of paired asteroids beyond $a=2.5$ AU.)

YORP fission. The second alternative for the origin of identified pairs is that they formed by the rotational fission of fast spinning asteroids. The radiative effect known as YORP (Yarkovsky-O'Keefe-Radzievski-Paddack effect; e.g., Bottke et al. 2006) may be the cause. The YORP effect can speed up or slow down asteroid rotation depending on surface properties of the small body and its obliquity, $\epsilon$ (Rubincam 2000; Vokrouhlický \& Čapek 2002; Čapek \& Vokrouhlický 2004). It has been observationally confirmed on asteroids (54509) YORP and (1862) Apollo (Lowry et al. 2007; Taylor et al. 2007; Kaasalainen et al. 2007).

A large fraction of small asteroids may be spun up by YORP beyond the cohesion strength threshold. If the identified pairs are indeed produced by the YORP-induced fission, we would expect that most of them should have, at least initially, nearly identical orbital inclinations. This is because the most common terminal spin states of the YORP-induced evolution have $\epsilon=0^{\circ}$ or $180^{\circ}$. Therefore, the fragments released by centrifugal force from the parent body should stay in the same orbital plane and have similar $i$ values. 
Interestingly, we found that the contributions of $\delta \sin i$ in Equation (1) for pairs with $d<10 \mathrm{~m} \mathrm{~s}^{-1}$ are generally negligible (relative to contributions from $\delta a$ and $\delta e$ ). This is in accord with the fact that for $d<10 \mathrm{~m} \mathrm{~s}^{-1}$, the $N(<d)$ distribution in Figure 1 is well approximated by $N(<d) \sim d^{2}$. The exponent 2 here means that a $2 \mathrm{D}$ subspace of $(a, e, i, \Omega, \varpi)$ dominates the value of $d$.

Moreover, as we described in Section 5, the encounter geometry of (6070) Rheinland and (54827) 2001 NQ8 about $17 \mathrm{kyr}$ ago was such that these two objects had a nearly zero speed component in the direction perpendicular to their orbit. These results may hint on the origin of paired asteroids. They are a feature that we would expect for asteroids disrupted by the YORP-induced fission. Photometric studies of the paired asteroid may be helpful to provide constraints on their current rotation states and possible spin histories.

Dissociation of binaries. The extremely low speed, $\approx 0.25 \mathrm{~m}$ $\mathrm{s}^{-1}$ (Section 5), during the $\approx 17 \mathrm{kyr}$ encounter between $(6070)$ Rheinland and (54827) 2001 NQ8 may be hinting on yet another formation process of the identified pairs. A large number of binaries has been identified among the main-belt and near-Earth asteroids (e.g., Merline et al. 2002; Pravec \& Harris 2007). The binaries with $\mathrm{km}$-sized components may be created and destabilized by radiation effects (Ćuk \& Burns 2005; Ćuk 2007; Bottke et al. 2006; Scheeres 2002, 2004, 2007). Moreover, a large number of binary systems is produced by catastrophic collisions (Durda et al. 2004; Nesvorný et al. 2006b) with many of them eventually dissolving due to dynamical instabilities. Therefore, the identified pairs may be binary systems that have become unbound. Low separation speeds, such as those of (6070) Rheinland and (54827) 2001 NQ8, would be expected in this case. Also, as we showed in Section 2, the mass ratio, $\mu$, of paired objects broadly matches that of the NEA binaries (Merline et al. 2002), which are thought to have formed by the YORP fission or disruptive collisions (e.g., Pravec et al. 2008; Durda et al. 2004). Therefore, dissolved NEA-like binaries by radiation effects or inherent dynamical instabilities are identified here as a possible formation mechanism of asteroid pairs.

We also note that the YORP fission, discussed above, and the binary dissociation may be closely related processes. Scheeres $(2002,2004,2007)$ showed that asteroids which spun into the fission limit are able to dissociate immediately, provided certain conditions on the mass distribution and shape morphology are satisfied. D. Scheeres (2008, private communication) has also shown that if a parent asteroid undergoes a dissociation due to gravitational instability, at least one of the fragments emerges from the process with a relatively slow rotation. Photometric observations could thus directly help to support or rule out this mechanism of the pair origin.

Additional considerations. Additional constraints on the pairs' origin may be derived from theoretical estimates of the efficiencies of the formation processes described above. We find that at least $\sim 10$ of pairs with $d<10 \mathrm{~m} \mathrm{~s}^{-1}$ correspond to a parent object with $D \geqslant 5 \mathrm{~km}$ (Figure 4 ). We estimate that $\sim 30$ such pairs exist in the whole main belt when the observational incompleteness is factored in. Using collisional modeling, Bottke et al. (2005a, 2005b) determined that one collisional disruption of a $D \geqslant 5 \mathrm{~km}$ asteroid happens in the main-belt asteroid, each $\sim 50$ kyr. Therefore, the estimated $\sim 30$ asteroid pairs could have been produced by disruptive collisions over $\sim 1.5 \mathrm{Myr}$. This is plausible because our results show that the orbit elements of the two objects in a pair typically remain similar over this timescale.
The YORP-induced rotational fission of $D \approx 1-5 \mathrm{~km}$ mainbelt asteroids may be a more efficient formation process than collisions. Based on the results of Čapek \& Vokrouhlický (2004), we estimate that a $D=5 \mathrm{~km}$ main-belt asteroid with a normal initial spin state could be spun up by YORP to the fission limit in a characteristic timescale of $\sim 100 \mathrm{Myr}$. If there are $\sim 10^{5}$ asteroids with $D \geqslant 5 \mathrm{~km}$ in the main belt (e.g., Bottke et al. 2005a, 2005b), we would expect that one $D \geqslant 5 \mathrm{~km}$ asteroid reaches the fission limit every $\sim 2 \mathrm{kyr}$ (assuming that $50 \%$ of asteroids are spun up by YORP). This made $\sim 50$ cases in the past $100 \mathrm{kyr}$. In addition, using the results of Ćuk \& Burns (2005) and Ćuk (2007), we estimate that a similar number of binaries could be destabilized in $100 \mathrm{kyr}$ by radiation effects. Therefore, if the YORP fission and/or binary dissociation are really as frequent as we estimate here, most of the identified pairs could be younger than $\sim 100 \mathrm{kyr}$. This is plausible based on our formation age estimates discussed in Section 4.

Paired asteroids represent an interesting population of the small main-belt objects and are prime targets for astronomical observations. Continuing astrometric observations will help to reduce the orbit uncertainty and thus improve our chances to estimate pairs' formation ages. Lightcurve observations will help to determine the shape and spin states of paired asteroids, including their rotation period and pole orientation. Observations of thermal radiation from these objects will improve our ability to constrain their size and surface thermal properties, such as the thermal conductivity. These results will help to get a better handle on the strength of the Yarkovsky effect on individual bodies. Consequently, using these constraints, $t_{\text {age }}$ could be potentially established for many pairs with a reasonable uncertainty, thus helping us to understand their formation. In addition, the surface age of the paired asteroids is likely to be $\lesssim 1$ Myr and for some, such as the case of (6070) Rheinland and (54827) 2001 NQ8, potentially younger than $\sim 50 \mathrm{kyr}$. Spectroscopic observations of these young objects could lead to significant results on asteroid composition and space weathering processes.

This research was supported by NASA's Planetary Geology and Geophysics Program (grant NAG513038), Czech Grant Agency (grant 205/08/0064), and the Research Program MSM0021620860 of the Czech Ministry of Education. Sergio Foglia and Maura Tombelli found numerous precoveries of paired asteroids, including the 2002 precovery of 2003 SQ168. This helped us to reduce orbit uncertainties. Andrea Boattini, Ed Beshore, and Rick Kowalski used a 60 inch telescope of the Catalina Sky Survey to obtain additional astrometry of 2004 TV14 in 2008 January. Luca Buzzi recovered 2003 SQ168 in 2007 December which led to the significant improvement of its orbit. Rob Matson and several other contributors to the Yahoo MPML discussion Web site independently discovered several asteroid pairs in 2007 December. We also thank Alan W. Harris (SSI), Petr Pravec, and Dan Scheeres for valuable discussions.

\section{REFERENCES}

Bendjoya, P., \& Zappalà, V. 2002, in Asteroids III, ed. W. F. Bottke et al. (Tucson, AZ: Univ. Arizona Press), 613

Bottke, W. F., Durda, D. D., Nesvorný, D., Jedicke, R., Morbidelli, A., Vokrouhlický, D., \& Levison, H. F. 2005a, Icarus, 175, 111

Bottke, W. F., Durda, D. D., Nesvorný, D., Jedicke, R., Morbidelli, A., Vokrouhlický, D., \& Levison, H. F. 2005b, Icarus, 179, 63 
Bottke, W. F., Vokrouhlický, D., Rubincam, D. P., \& Nesvorný, D. 2006, Annu. Rev. Earth Planet. Sci., 34, 157

Bowell, E., Muinonen, K., \& Wasserman, L. H. 1994, in Asteroids, Comets and Meteors, ed. A. Milani, M. di Martino, \& A. Cellino (Dordrecht: Kluwer Academic), 477

Čapek, D., \& Vokrouhlický, 2004, Icarus, 172, 526

Ćuk, M. 2007, ApJ, 659, 57

Cuk, M., \& Burns, J. A. 2005, Icarus, 176, 418

Durda, D. D., Bottke, W. F., Enke, B. L., Merline, W. J., Asphaug, E., Richardson, D. C., \& Leinhardt, Z. M. 2004, Icarus, 167, 382

Durda, D. D., Bottke, W. F., Nesvorný, D., Enke, B. L., Merline, W. J., Asphaug, E., \& Richardson, D. C. 2007, Icarus, 186, 498

Hirayama, K. 1918, AJ, 31, 185

Ivezić, Z., et al. 2001, AJ, 122, 2749

Jedicke, R., Magnier, E. A., Kaiser, N., \& Chambers, K. C. 2007, in Near Earth Objects, our Celestial Neighbors: Opportunity and Risk, ed. A. Milani et al. (Cambridge, MA: Cambridge Univ. Press), 341

Kaasalainen, M., Ďurech, J., Warner, B. D., Krugly, Y. N., \& Gaftonyuk, N. M. 2007, Nature, 446, 420

Knežević, Z., Lemaitre, A., \& Milani, A. 2002, in Asteroids III, ed. W. F. Bottke et al. (Tucson, AZ: Univ. Arizona Press), 603

Levison, H. F., \& Duncan, M. J. 1994, Icarus, 108, 18

Lowry, S. C., et al. 2007, Science, 316, 272
Merline, W. J., Weidenschilling, S. J., Durda, D. D., Margot, J. L., Pravec, P. \& Storrs, A. D. 2002, in Asteroids III, ed. W. F. Bottke et al. (Tucson, AZ: Univ. Arizona Press), 289

Nesvorný, D., \& Bottke, W. F. 2004, Icarus, 170, 324

Nesvorný, D., Bottke, W. F., Dones, L., \& Levison, H. F. 2002, Nature, 417, 720

Nesvorný, D., Bottke, W. F., Levison, H. F., \& Dones, L. 2003, ApJ, 591, 486

Nesvorný, D., Enke, B. L., Bottke, W. F., Durda, D. D., Asphaug, E., \& Richardson, D. C. 2006b, Icarus, 183, 296

Nesvorný, D., Jedicke, R., Whiteley, R. J., \& Ivezić, Ž. 2005, Icarus, 173, 132

Nesvorný, D., \& Vokrouhlický, D. 2006, AJ, 132, 1950

Nesvorný, D., Vokrouhlický, D., \& Bottke, W. F. 2006a, Science, 312, 1490

Pravec, P., \& Harris, A. W. 2007, Icarus, 190, 250

Pravec, P., et al. 2008, Icarus, in press

Rubincam, D. P. 2000, Icarus, 148, 2

Scheeres, D. 2002, Celest. Mech. Dyn. Astron., 83, 155

Scheeres, D. 2004, Celest. Mech. Dyn. Astron., 89, 127

Scheeres, D. 2007, Icarus, 189, 370

Taylor, P. A., et al. 2007, Science, 316, 274

Vokrouhlický, D. 1999, A\&A, 344, 362

Vokrouhlický, D., \& Capek, D. 2002, Icarus, 159, 449

Vokrouhlický, D., Čapek, D., Chesley, S. R., \& Ostro, S. J. 2005, Icarus, 179, 128

Vokrouhlický, D., Milani, A., \& Chesley, S. R. 2000, Icarus, 148, 118

Zappalà, V., Cellino, A., Farinella, P., \& Knežević, Z. 1990, AJ, 100, 2030 